\title{
Erratum: the local potential approximation in quantum gravity
}

\section{Dario Benedetti ${ }^{a}$ and Francesco Caravelli ${ }^{a, b, c}$}

${ }^{a}$ Max Planck Institute for Gravitational Physics (Albert Einstein Institute),

Am Mühlenberg 1, D-14476 Golm, Germany

${ }^{b}$ Perimeter Institute for Theoretical Physics,

Waterloo, Ontario N2L $2 Y 5$ Canada

${ }^{c}$ University of Waterloo,

Waterloo, Ontario N2L 3G1, Canada

E-mail: dario.benedetti@aei.mpg.de, fcaravelli@perimeterinstitute.ca

ERRATUM TO: JHEP06(2012)017

KeYwords: Models of Quantum Gravity, Renormalization Group

Equation (5.7) in the original paper contains a mistake, and it should be corrected to

$$
\begin{aligned}
W_{0}^{\bar{h}}(z, \tilde{R})= & \frac{3\left(1-z^{2}\right)\left(3 \partial_{t} \tilde{f}^{\prime \prime}(\tilde{R})-6 \tilde{R} \tilde{f}^{(3)}(\tilde{R})\right)}{18 \tilde{f}^{\prime \prime}(\tilde{R})-2(\tilde{R}-3) \tilde{f}^{\prime}(\tilde{R})+4 \tilde{f}(\tilde{R})} \\
& +\frac{3(1-z)\left(\partial_{t} \tilde{f}^{\prime}(\tilde{R})-2 \tilde{R} \tilde{f}^{\prime \prime}(\tilde{R})+2 \tilde{f}^{\prime}(\tilde{R})\right)+6\left(\tilde{f}^{\prime}(\tilde{R})+6 \tilde{f}^{\prime \prime}(\tilde{R})\right)}{18 \tilde{f}^{\prime \prime}(\tilde{R})-2(\tilde{R}-3) \tilde{f}^{\prime}(\tilde{R})+4 \tilde{f}(\tilde{R})} .
\end{aligned}
$$

The error propagates to sections 6 and 7 , affecting part of the numerical results, but not the qualitative aspects. In particular, equation (6.5) should be

$$
\begin{aligned}
& \mathcal{T}_{0}^{\bar{h}}=\frac{1}{2 \tilde{R}^{2}\left(-9 \tilde{f}^{\prime \prime}(\tilde{R})+(\tilde{R}-3) \tilde{f}^{\prime}(\tilde{R})-2 \tilde{f}(\tilde{R})\right)} \times \\
& \left\{\left(\tilde{R}^{4}-54 \tilde{R}^{2}-54\right)\left(\partial_{t} \tilde{f}^{\prime \prime}(\tilde{R})-2 \tilde{R} \tilde{f}^{(3)}(\tilde{R})\right)\right. \\
& -\left(\tilde{R}^{3}+18 \tilde{R}^{2}+12\right)\left(\partial_{t} \tilde{f}^{\prime}(\tilde{R})-2 \tilde{R} \tilde{f}^{\prime \prime}(\tilde{R})+2 \tilde{f}^{\prime}(\tilde{R})\right) \\
& \left.-36\left(\tilde{R}^{2}+2\right)\left(\tilde{f}^{\prime}(\tilde{R})+6 \tilde{f}^{\prime \prime}(\tilde{R})\right)\right\} .
\end{aligned}
$$

Equations (6.7) and (7.6) should be corrected accordingly, as well as the numerical results in sections 7.4 and 7.5. All the qualitative aspects of the results (e.g. section 7.6), as well as the general assessment and conclusions, are unaffected by such corrections. 